\title{
Pointwise convergence rates and central limit theorems for kernel density estimators in linear processes
}

\author{
Anton Schick* \\ Binghamton University
}

\author{
Wolfgang Wefelmeyer \\ Universität zu Köln
}

\begin{abstract}
Convergence rates and central limit theorems for kernel estimators of the stationary density of a linear process have been obtained under the assumption that the innovation density is smooth (Lipschitz). We show that smoothness is not required. For example, it suffices that the innovation density has bounded variation.
\end{abstract}

Keywords. Lipschitz continuity, martingale approximation, smoothness of convolutions.

\section{Introduction}

Consider a linear process $X_{t}=\sum_{s=0}^{\infty} a_{s} \varepsilon_{t-s}$ with independent and identically distributed (i.i.d.) innovations $\varepsilon_{t}$ that have mean zero, finite variance, and density $f$. We assume that $a_{0}=1$ and that the coefficients are summable, $\sum_{s=0}^{\infty}\left|a_{s}\right|<\infty$. Then $X_{t}$ has a stationary density $g$. It can be estimated by the kernel estimator

$$
\hat{g}(x)=\frac{1}{n} \sum_{j=1}^{n} k_{b}\left(x-X_{j}\right),
$$

where $k_{b}(v)=k(v / b) / b$ with $k$ a square-integrable kernel (i.e. a function such that $\int k(v) d v=1$ and $\left.\int k^{2}(v) d v<\infty\right)$, and $b$ is a bandwidth such that $b \rightarrow 0$ and $n b \rightarrow \infty$. Pointwise and uniform convergence rates have been studied by several authors, see for example Hall and Hart (1990), Tran (1992), Hallin and Tran (1996), Lu (2001), Wu and Mielniczuk (2002), and Bryk and Mielniczuk (2005).

$\mathrm{Wu}$ and Mielniczuk (2002) prove the following result: If $f$ is Lipschitz, then $\sqrt{n b}(\hat{g}(x)-$ $E[\hat{g}(x)])$ is asymptotically normal with mean zero and variance $\sigma^{2}(x)=g(x) \int k^{2}(v) d v$. We prove in Section 2 that this result can be obtained under weaker assumptions on $f$. In particular, it suffices that $f$ is of bounded variation, except in the degenerate case with $a_{s}=0$ for all $s \geq 1$. This exceptional case corresponds to the i.i.d. case, for which sufficient conditions are already known, see Parzen (1962). In the latter case moment assumptions are not needed. Instead Parzen requires continuity of $f=g$ at $x$ and a kernel that is bounded and satisfies $|v k(v)| \rightarrow 0$ as $|v| \rightarrow \infty$.

\footnotetext{
* Supported in part by NSF Grant DMS 0405791
} 


\section{Results}

We consider observations $X_{1}, \ldots, X_{n}$ of a linear process with the following properties.

Assumption 1. The process $X_{t}, t \in \mathbb{Z}$, has the representation $X_{t}=\sum_{s=0}^{\infty} a_{s} \varepsilon_{t-s}$ with i.i.d. innovations $\varepsilon_{t}$ that have mean zero, finite variance, and density $f$. The coefficients $a_{s}, s \in \mathbb{N}$, are summable, $a_{0}=1$, and $N$ is positive, where

$$
N=\sum_{s=1}^{\infty} \mathbf{1}\left(a_{s} \neq 0\right)
$$

denotes the number of non-zero coefficients $a_{s}, s \geq 1$. The kernel $k$ is square-integrable and the bandwidth satisfies $b \rightarrow 0$ and $n b \rightarrow \infty$.

We restrict ourselves to the case $N>0$, since $N=0$ corresponds to the i.i.d. case already treated by Parzen (1962).

Let $g$ denote the stationary density and $\hat{g}$ the kernel estimator described above. First we obtain the above-mentioned conclusion of Theorem 1 in Wu and Mielniczuk (2002) under weaker assumptions. We do this by generalizing their approach. They express $\hat{g}(x)-E[\hat{g}(x)]=$ $\hat{g}(x)-g * k_{b}(x)$ as a sum $D_{0}+R_{0}$, where

$$
D_{0}=\frac{1}{n} \sum_{j=1}^{n}\left(k_{b}\left(x-X_{j}\right)-f * k_{b}\left(x-Y_{j, 1}\right)\right)
$$

is a martingale, and

$$
R_{0}=\frac{1}{n} \sum_{j=1}^{n}\left(f * k_{b}\left(x-Y_{j, 1}\right)-g * k_{b}(x)\right) .
$$

Here we use the notation

$$
Y_{j, i}=\sum_{s=i}^{\infty} a_{s} \varepsilon_{j-s} .
$$

Then they show in their Lemma 2 that $\sqrt{n b} D_{0} \Rightarrow N\left(0, \sigma^{2}(x)\right)$ under the assumption that $f$ is Lipschitz. In their proof, this assumption is used only to guarantee continuity of $g$ at $x$, boundedness of $f$ and hence $g$, and to prove

$$
\int k^{2}(v)|\bar{H}(b v)-\bar{H}(0)| d v=o_{p}(1)
$$

where

$$
\bar{H}(z)=\frac{1}{n} \sum_{j=1}^{n}\left(f\left(x-Y_{j, 1}-z\right)-g(x-z)\right) .
$$

Assume now that $f$ is bounded. This guarantees that $g$ is bounded and uniformly continuous. Indeed, we can express $g$ as the convolution of $f$ and the density $g_{1}$ of $Y_{1,1}$ and obtain $g(x) \leq$ $\|f\|_{\infty}\left\|g_{1}\right\|_{1}$ and

$$
|g(x-t)-g(x)| \leq\|f\|_{\infty} \int\left|g_{1}(y-t)-g_{1}(y)\right| d y
$$


for all $x \in \mathbb{R}$. Uniform continuity of $g$ now follows from the continuity of translations in $L_{1}$, see e.g. Rudin (1974, Theorem 9.5). In view of these properties of $g$ and the square-integrability of $k$, property (2.1) follows from

$$
\int k^{2}(v)\left|\frac{1}{n} \sum_{j=1}^{n}\left(f\left(x-Y_{j, 1}-b v\right)-f\left(x-Y_{j, 1}\right)\right)\right| d v=o_{p}(1) .
$$

The expected value of the left-hand side of (2.2) is bounded by

$$
\begin{array}{r}
\int k^{2}(v) \int|f(x-y-b v)-f(x-y)| g_{1}(y) d y d v \\
\leq\left\|g_{1}\right\|_{\infty} \int k^{2}(v) \int|f(t-b v)-f(t)| d t d v .
\end{array}
$$

Since $\int|f(t-b v)-f(t)| d t$ is bounded by 2 and tends to zero by the continuity of translations in $L_{1}$, the Lebesgue dominated convergence theorem gives that

$$
\int k^{2}(v) \int|f(t-b v)-f(t)| d t d v \rightarrow 0 .
$$

Thus the conclusion of their Lemma 2 remains true if $f$ is bounded.

Wu and Mielniczuk (2002) also use Lipschitz continuity of $f$ to conclude that $\sqrt{n} R_{0}=O_{p}(1)$. To avoid this assumption, we express $R_{0}$ as

$$
R_{0}=\sum_{i-1}^{m} D_{i}+R_{m}
$$

where

$$
\begin{aligned}
D_{i} & =\frac{1}{n} \sum_{j=1}^{n}\left(f_{i-1} * k_{b}\left(x-Y_{j, i}\right)-f_{i} * k_{b}\left(x-Y_{j, i+1}\right)\right), \\
R_{m} & =\frac{1}{n} \sum_{j=1}^{n}\left(f_{m} * k_{b}\left(x-Y_{j, m+1}\right)-g * k_{b}(x)\right) .
\end{aligned}
$$

Here $f_{i}$ is the density of $\sum_{s=0}^{i} a_{s} \varepsilon_{s}$. Then $f_{i+1}(x)=\int f_{i}\left(x-a_{i+1} y\right) f(y) d y$ and $f_{0}=f$. For $i=1,2, \ldots$, the sequence $D_{i}$ is a square-integrable martingale with

$$
\begin{aligned}
n E\left[D_{i}^{2}\right] & \leq E\left[\left(f_{i-1} * k_{b}\right)^{2}\left(x-Y_{1, i}\right)\right] \\
& \leq\left\|f_{i-1} * k_{b}\right\|_{\infty} E\left[f_{i-1} *\left|k_{b}\right|\left(x-Y_{1, i}\right)\right] \\
& \leq\left\|f_{i-1}\right\|_{\infty} \int|k(v)| d v g *\left|k_{b}\right|(x) \\
& \leq\left(\|f\|_{\infty} \int|k(v)| d v\right)^{2} .
\end{aligned}
$$

Thus, if $f$ is bounded, then $\sqrt{n} D_{i}=O_{p}(1)$.

Now consider $R_{m}$. If $a_{s}=0$ for all $s>m$, then $Y_{j, m+1}=0$ and thus $R_{m}=0$. Now assume that there are infinitely many $a_{s}$ that are not zero, and that $f_{m}$ is Lipschitz. Then we can 
proceed as in the proofs of Lemma 3 (with our $f_{m}$ replacing their $f_{1}$ ) and Theorem 1 of Wu and Mielniczuk (2002) to conclude that $\sqrt{n} R_{m}=O_{p}(1)$. We arrive at the following result.

Theorem 1. Suppose Assumption 1 holds and $f$ is bounded. If $N=\infty$, assume also that $f_{m}$ is Lipschitz for some $m$. Then

$$
\sqrt{n b}\left(\hat{g}(x)-g * k_{b}(x)\right) \Rightarrow N\left(0, \sigma^{2}(x)\right)
$$

where $\sigma^{2}(x)=g(x) \int k^{2}(v) d v$.

Remark 1. A function $h$ is $L_{1}$-Lipschitz if

$$
\int|f(x-t)-f(x)| d x \leq L|t|, \quad t \in \mathbb{R}
$$

for some constant $L$. If $N=\infty$, the requirement that $f_{m}$ is Lipschitz for some $m$ can be replaced by the requirement that $f_{r}$ is $L_{1}$-Lipschitz for some $r$. To see this, let $m=\inf \left\{s>r: a_{s} \neq 0\right\}$. Then $f_{m}(x)=\int f_{r}\left(x-a_{m} y\right) f(y) d y$. Since $f$ is assumed bounded, we have

$$
\begin{aligned}
\left|f_{m}(x+t)-f_{m}(x)\right| & \leq \int\left|f_{r}\left(x+t-a_{m} z\right)-f_{r}\left(x-a_{m} z\right)\right| f(z) d z \\
& \leq\|f\|_{\infty} \int\left|f_{r}\left(x+t-a_{m} z\right)-f_{r}\left(x-a_{m} z\right)\right| d z \\
& \leq\|f\|_{\infty} \frac{1}{\left|a_{m}\right|} \int\left|f_{r}(u+t)-f_{r}(u)\right| d u \\
& \leq\|f\|_{\infty} \frac{1}{\left|a_{m}\right|} L|t| .
\end{aligned}
$$

The requirement that a density is $L_{1}$-Lipschitz is rather mild: it is met by all densities of bounded variation. For details see Lemma 8 in Schick and Wefelmeyer (2005). Thus, if $f$ is of bounded variation, we have the following result.

Corollary 1. Suppose Assumption 1 holds and $f$ is of bounded variation. Then

$$
\sqrt{n b}\left(\hat{g}(x)-g * k_{b}(x)\right) \Rightarrow N\left(0, \sigma^{2}(x)\right) .
$$

So far we have looked at the variance term $\hat{g}(x)-g * k_{b}(x)$. The bias term $g * k_{b}(x)-g(x)$ can be made small by choice of $b$ and $k$ if $g$ is sufficiently smooth. Smoothness of $g$ does not require smoothness of $f$ if $N>0$. For example, if $f$ is of bounded variation and $N \geq m>0$, then $g$ has $m-1$ bounded derivatives, and $g^{(m-1)}$ is Lipschitz. This follows by induction from Lemma 1 below. Thus we have the following theorem.

Theorem 2. Suppose Assumption 1 holds. If $f$ is of bounded variation, a kernel of order $m$ is used, and $N \geq m$, then

$$
\hat{g}(x)-g(x)=O_{p}\left(n^{-1 / 2} b^{-1 / 2}\right)+O\left(b^{m}\right) .
$$


Remark 2. Suppose $N$ is known to be infinite and $f$ has bounded variation. Then we can control the rate $O\left(b^{m}\right)$ of the bias by choosing a kernel of high order $m$. A choice of bandwidth $b \sim n^{-1 /(2 m+1)}$ yields the rate $\hat{g}(x)-g(x)=O_{p}\left(n^{-m /(2 m+1)}\right)$. Thus we can achieve a rate close to the parametric rate $n^{-1 / 2}$.

\section{An Auxiliary Result}

Lemma 1. Let $p$ and $q$ be integrable functions with $q$ of bounded variation and $p$ bounded and $L_{1}$-Lipschitz. Then $p * q$ is Lipschitz and has an almost everywhere derivative that is bounded, integrable, and $L_{1}$-Lipschitz.

Proof. The proof is similar to the proof of Lemma 8 in Schick and Wefelmeyer (2005). Since $q$ is integrable and of bounded variation, we can write $q$ as difference of two bounded nondecreasing functions that vanish at $-\infty$. Without loss of generality we may assume that these functions are right-continuous, as this changes $q$ only on a countable set and hence does not affect $p * q$. Thus we may assume that

$$
q(x)=\mu_{1}(-\infty, x]-\mu_{2}(-\infty, x], \quad x \in \mathbb{R},
$$

for two finite measures $\mu_{1}$ and $\mu_{2}$. Let $\mu=\mu_{1}+\mu_{2}$ and set $h=h_{1}-h_{2}$, where

$$
h_{i}(x)=\int p(x-y) \mu_{i}(d y), \quad x \in \mathbb{R}, \quad i=1,2 .
$$

Note that $h$ is integrable and bounded since

$$
\left\|h_{i}\right\|_{1} \leq \iint|p(x-y)| \mu_{i}(d y) d x \leq\|p\|_{1} \mu_{i}(\mathbb{R}) \quad \text { and } \quad\left\|h_{i}\right\|_{\infty} \leq\|p\|_{\infty} \mu_{i}(\mathbb{R}), \quad i=1,2 .
$$

We have

$$
\begin{aligned}
\int_{-\infty}^{z} h_{i}(x) d x & =\iint_{x \leq z} p(x-y) d x \mu_{i}(d y) \\
& =\iint_{u \leq z-y} p(u) d u \mu_{i}(d y) \\
& =\int \mu_{i}(-\infty, z-u] p(u) d u .
\end{aligned}
$$

This shows that $p * q$ is absolutely continuous with almost everywhere derivative $h$. As $h$ is bounded, $p * q$ is Lipschitz. In view of the inequality

$$
\begin{aligned}
\int|h(x+t)-h(x)| d x & \leq \iint|p(x+t-y)-p(x-y)| d x \mu(d y) \\
& \leq \mu(\mathbb{R}) \int|p(x+t)-p(x)| d x
\end{aligned}
$$

the function $h$ inherits the $L_{1}$-Lipschitz property from $p$. 


\section{References}

Bryk, A. and Mielniczuk, J. (2005), Asymptotic properties of density estimates for linear processes: application of projection method, J. Nonparametr. Stat. 17, 121-133.

Hall, P. and Hart, J. D. (1990), Convergence rates in density estimation for data from infinite-order moving average processes, Probab. Theory Related Fields 87, 253-274.

Hallin, M. and Tran, L. T. (1996), Kernel density estimation for linear processes: Asymptotic normality and optimal bandwidth derivation, Ann. Inst. Statist. Math. 48, 429-449.

Lu, Z. (2001), Asymptotic normality of kernel density estimators under dependence, Ann. Inst. Statist. Math. 53, 447-468.

Parzen, E. (1962), On estimation of a probability density function and mode, Ann. Math. Statist. 33, $1065-1076$.

Rudin, W. (1974), Real and Complex Analysis (McGraw-Hill, New York, 2nd ed.).

Schick, A. and Wefelmeyer, W. (2005), Root- $n$ consistent density estimators of convolutions in weighted $L_{1}$ norms, Technical Report, Department of Mathematical Sciences, Binghamton University.

Tran, L. T. (1992), Kernel density estimation for linear processes, Stochastic Process. Appl. 41, 281-296.

Wu, W. B. and Mielniczuk, J. (2002), Kernel density estimation for linear processes, Ann. Statist. 30, 1441-1459.

\author{
ANTON SCHICK \\ Department of Mathematical Sciences \\ BINGHAMTON UNIVERSITY \\ Binghamton, NY 13902-6000 \\ USA \\ ANTON@MATH . BINGHAMTON . EDU \\ HTTP : //MATH . BINGHAMTON. EDU/ANTON/
}

\author{
WOLFGANG WEFELMEYER \\ MATHEMATISChES Institut \\ UNIVERSITÄT ZU KÖLN \\ 50931 KÖLN \\ GERMANY \\ WEFELMOMATH . UN I-KOELN . DE \\ HTTP: //WWW.MI. UNI-KOELN.DE/ WEFELM/
}

